\title{
Determining the Expressions of Cytokeratin 5/6 by Immunohistochemistry in Basal Like Triple Negative Breast Carcinoma and Its Correlation with Histomorphological Grade
}

Dr. Nure Jannat Ferdousi Ara ${ }^{1 *}$, Dr. Md Mustakim Ali $^{2}$, Dr. Asaduzzaman ${ }^{3}$, Dr. Monica Mehjabin ${ }^{4}$, Professor Dr. Ferdousy Begum ${ }^{5}$

\author{
${ }^{1}$ Assistant professor, Department of Pathology, M Abdur Rahim Medical College Hospital, Dinajpur, Bangladesh \\ ${ }^{2}$ Assistant professor, Dept of Paediatrics, M Abdur Rahim Medical College, Dinajpur, Bangladesh \\ ${ }^{3}$ Assistant professor, Pathology, Sheikh Hasina National Institute of Burn \& plastic surgery, Dhaka, Bangladesh \\ ${ }^{4}$ Lecturer, Dept. of Pathology, Shaheed Suhrawardy Medical College, Dhaka, Bangladesh \\ ${ }^{5}$ Professor, Department of Pathology, Bangabandhu Sheikh Mujib Medical University (BSMMU), Dhaka, Bangladesh
}

DOI: $10.36347 /$ sasjm.2021.v07i05.010

| Received: 05.03.2021 | Accepted: 06.04.2021 | Published: 30.05.2021

*Corresponding author: Nure Jannat Ferdousi Ara

Introduction: Breast cancer is the most commonly happening female cancer and the leading cause of cancer deaths worldwide. Breast cancer is a heterogeneous disease and it encompasses a variety of entities with distinct morphological appearances and clinical behaviors. Triple-negative breast cancer (TNBC) represents $12.5-15 \%$ of all breast cancers. Its molecular dissimilarity also brings a significantly different clinical course. It has been observed that basal-like breast cancers exhibit a gene expression profile very similar to that of myoepithelial cells. Aim of the study: To identify the basal like TNBC cases and correlate them with histomorphological grade. Material \& Methods: This cross sectional study was conducted in the department of BSMMU from January 2016 to December 2017. A total of 41 cases previously diagnosed as TNBC by histological and immunohistochemical examinations were selected for the study. During collecting specimen, all relevant information were recorded systemically in a prescribed proforma. Histopathogical type of tumor was done according to WHO classification of breast tumor, 2012 and grading was done followings Nottingham modification of the Bloom -Richardson Grading System, and recorded. Immunohistochemistry of ER, PR and HER-2/neu expression of all cases were performed using Dako Autostainer Plus at the immunohistochemistry laboratory, of department of Pathology, BSMMU. Statistical analyses of the results were obtained by window based computer software devised with Statistical Packages for Social Sciences (SPSS-20). Prior to the commencement of this study the thesis protocol was submitted to the Institutional Review Board (IRB) of BSMMU, Dhaka for approval and was approved. Results: A total number of 41 cases were selected for the study out of these 41 cases; most of the cases belong to a range of 31 to 40 years. Minimum age was 26 years, maximum age was 70 years. The mean age was 42 . There is no association between age and CK 5/6 immunoreactivity. It is not statistically significant at $\alpha=0.05$ level. Out of 41 cases $1(2 \%)$ case was grade $1,24(59 \%)$ cases were grade 2 and 16 $(39 \%)$ cases were grade 3 . According to the grade and the status of the membrane /cytoplasmic expression of CK $5 / 6$ protein, grade I, grade II and grade III tumors show 00(00\%), 14(53\%) and 12(46\%) expression of CK5/6 respectively. It shows the distribution of cases according to the expression of CK5/6 and their relation with grades .It appears that percentage of CK5/6 expression is high in high grade tumor. Conclusion: Triple-Negative Breast Cancer' encompasses a heterogeneous group of tumors that possess distinctive pathological and clinical features. CK5/6 expression could serve as biomarker for identifying TNBC patients with poor survival. These patients are not benefited from neoadjuvant chemotherapy.

Keywords: Breast Cancer, Immunohistochemistry, Histomorphological, Expressions, Cytokeratin 5/6.

Copyright $(\mathcal{C} 2021$ The Author(s): This is an open-access article distributed under the terms of the Creative Commons Attribution 4.0 International License (CC BY-NC 4.0) which permits unrestricted use, distribution, and reproduction in any medium for non-commercial use provided the original author and source are credited.

\section{INTRODUCTION}

Breast cancer is the most commonly happening female cancer and the leading cause of cancer deaths worldwide. Breast cancer is a heterogeneous disease and it encompasses a variety of entities with distinct morphological appearances and clinical behaviors. Triple-negative breast cancer (TNBC) represents 12.5 $15 \%$ of all breast cancers. Its molecular dissimilarity 
also brings a significantly different clinical course. The outcome of this cancer is much more aggressive. Very often already at the moment of diagnosis the presence of distant metastases is ascertained. Many risk factors for breast cancer have been defined. The most important are female sex, older age, early age at first menstruation, lack of physical exercise, late age of the last menstrual period, long-term hormone replacement therapy, history of exposure to ionizing radiation, high body mass index (BMI) in young women. In such cases median survival is only one year. Very characteristic of TNBC is also absence of an association between the size of the primary tumor and the presence of metastases in the regional lymph nodes. It was necessary to establish a more detailed classification of lesions belonging to the triple-negative subgroup because molecular studies showed high variability within this type. $75-80 \%$ of tumors classified as TNBCs belong to the basal-like breast cancer group. The BLBC subtype is characterized by a particularly high histological grade, high mitotic index and low differentiation. Most commonly these tumors are described as anaplastic and metaplastic lesions. One of the reasons for worse prognosis is the high neovascularization level caused by vascular endothelial growth factor (VEGF) overexpression [1]. Basal-like Triple Negative Breast Carcinoma (TNBC) has increasing attention due to overall poor prognosis even after therapy. A panel of four antibodies (ER, HER1, HER-2, and cytokeratin 5/6) can accurately identify basal-like TNBC. Great importance is also attributed to genetic factors. Among them, carrier status of the BRCA1, BRCA2, TP53, PTEN and CHEK2 genes seems to be significant. The lifetime risk of breast cancer in women with a mutation in the BRCA1 or BRCA2 gene is $60-80 \%$. The basic method used in diagnosis of breast cancer is histopathological assessment. Evaluation of estrogen receptor (ER), progesterone receptor $(\mathrm{PgR})$ and human epidermal growth factor receptor 2 (HER2) expression is a standard diagnostic procedure. Immunohistochemical staining makes it possible to assess and allows to determine patient's prognosis much more properly and establish the most appropriate method of treatment. It has been observed that basal-like breast cancers exhibit a gene expression profile very similar to that of myoepithelial cells. However, it has been concluded that BLBCs show an incomplete phenotype of myoepithelial cells. Most frequently they show CK5/6 and EGFR expression with concomitant lack of SMA, CD10 and p63 expression.

\section{METHODOLOGY AND MATERIALS}

This cross sectional study was conducted in the department of BSMMU from January 2016 to December 2017. A total of 41 cases previously diagnosed as TNBC by histological and immunohistochemical examinations were selected according to following inclusion and exclusion criteria for the study. During collecting specimen, all relevant information were recorded systemically in a prescribed proforma. Aim of the study was to identify the basal like TNBC cases and correlate them with histomorphological grade. Histopathogical type of tumor was done according to WHO classification of breast tumor, 2012 and grading was done followings Nottingham modification of the Bloom -Richardson Grading System, and recorded. Immunohistochemistry of ER, PR and HER-2/neu expression of all cases were performed using Dako Autostainer Plus at the immunohistochemistry laboratory, of department of Pathology, BSMMU. Scoring were done by multiplying the percentage of $\mathrm{CK} 5 / 6$ expression cells with the intensity of its positivity. Tumors were classified based on positivity and negativity of the stain. No staining observed or membrane staining is observed in less than $10 \%$ of tumor cells. A faint/barely perceptible membrane staining is detected in more than $10 \%$ of tumor cells. A weak to moderate complete or incomplete membrane staining is seen in $>10 \%$ of tumor cells (weakly positive). A strong complete membrane staining is seen in $>30 \%$ of tumor cells (strongly positive). Scoring were done by multiplying the percentage of $\mathrm{CK} 5 / 6$ expression cells with the intensity of its positivity. Then the scores of percentage and staining intensity were multiplied to produce a weighted score for each tumor. A score of less than four (2-3) was considered as low expression and more than the four was considered as high expression. Statistical analyses of the results were obtained by window based computer software devised with Statistical Packages for Social Sciences (SPSS-20). Prior to the commencement of this study the thesis protocol was submitted to the Institutional Review Board (IRB) of BSMMU, Dhaka for approval and was approved.

\section{Inclusion Criteria}

- Breast tumor samples were taken from paraffin blocks diagnosed as triple-negative invasive breast carcinoma by histopathology and immunohistochemistry.

\section{Exclusion Criteria}

- Inadequate tissue present in paraffin blocks.

- Equivocal results.

- Recurrence breast carcinoma cases.

- Cases treated with neoadjuvant chemotherapy or radiotherapy prior to surgery.

\section{RESULTS}

A total number of 41 cases were selected for the study out of these 41 cases; most of the cases belong to a range of 31 to 40 years. Minimum age was 26 years, maximum age was 70 years. The mean age was 42 (Table-1). This table shows that, there is no association between age and CK 5/6 immunoreactivity. It is not statistically significant at $\alpha=0.05$ level (Table$2)$. Out of 41 cases 1 (2\%) case was grade 1, $24(59 \%)$ cases were grade 2 and $16(39 \%)$ cases were grade 3 . It appears that maximum cases belong to grade-II (Table- 
3). Total number of cases was 41 . Among them, 1 case was grade-1, 24 cases were grade -II and 16 cases were grade-III. Number of $\mathrm{CK} 5 / 6$ positive case was ' 0 '(zero) and negative was I (one) in grade-1, number of positive cases were 14 and negative were 10 in grade-II and number of positive cases were 12 and negative cases were 4 in grade-III. It appears that gradeI tumor is negative and only the high grade cases shows positivity (Table-4). According to the grade and the status of the membrane /cytoplasmic expression of CK $5 / 6$ protein, grade I, grade II and grade III tumors show $00(00 \%), 14(53 \%)$ and $12(46 \%)$ expression of CK5/6 respectively. After the statistical analysis by SPSS 20 Spearman rank correlation coefficient was 0.99 . T value was 43.84 with a 39 degree of freedom, $(\mathrm{df}=\mathrm{n}-2), \mathrm{P}$ value is $<0.05$. It shows the distribution of cases according to the expression of CK5/6 and their relation with grades .It appears that percentage of CK5/6 expression is high in high grade tumor (Table-5).

Table-1: Age distribution of TNBC cases.

\begin{tabular}{|l|l|l|}
\hline Age(in years) & Number of patient & Percentage \\
\hline $20-30$ & 5 & $12 \%$ \\
\hline $31-40$ & 14 & $34 \%$ \\
\hline $41-50$ & 11 & $26 \%$ \\
\hline $51-60$ & 9 & $21 \%$ \\
\hline $61-70$ & 2 & $4 \%$ \\
\hline
\end{tabular}

Table-2: Association between CK5/6 immunoreactivity and age distribution of TNBC case.

\begin{tabular}{|l|l|l|l|l|l|}
\hline \multicolumn{2}{l}{$\begin{array}{l}\text { Clinicopathological } \\
\text { parameter }\end{array}$} & No of cases & \multicolumn{2}{|l|}{ CK 5/6 immunoreactivity of total 41 cases } & P value \\
\cline { 3 - 5 } & & Positive & Negative & 3.34 (critical value) P > \\
\cline { 1 - 4 } Age (years) & $\geq 40$ & $23(56 \%)$ & $12(29 \%)$ & $11(26 \%)$ & \\
\cline { 2 - 5 } & $<40$ & $18(43 \%)$ & $14(34 \%)$ & $4(10 \%)$ & \\
\hline
\end{tabular}

Table-3: Distribution of patients according to tumor grade.

\begin{tabular}{|l|l|l|}
\hline Grade & Case & Percentage \\
\hline Grade-I & 1 & $2 \%$ \\
\hline Grade-II & 24 & $59 \%$ \\
\hline Grade-III & 16 & $39 \%$ \\
\hline
\end{tabular}

Table-4: Distribution of CK 5/6 positive and negative status in each grade.

\begin{tabular}{|l|l|l|}
\hline CK5/6 Grade & Positive CK5/6 & Negative CK5/6 \\
\hline Grade I- (1) & 0 & 1 \\
\hline Grade II- (24) & 14 & 10 \\
\hline Grade III- (16) & 12 & 4 \\
\hline
\end{tabular}

Table-5: Correlation between tumor grade with low and high expression of CK 5/6.

\begin{tabular}{|l|l|l|}
\hline Tumor grade & Low expression of CK 5/6 & High expression of CK 5/6 \\
\hline Grade 1 & $00(00 \%)$ & $00(00 \%)$ \\
\hline Grade 2 & $10(38 \%)$ & $4(15 \%)$ \\
\hline Grade 3 & $5(19 \%)$ & $7(27 \%)$ \\
\hline
\end{tabular}

\section{DISCUSSION}

Out of the total 41cases $26(63.41 \%)$ cases showed positive Cytokeratin staining. Number of Cytokeratin positive cases was highest in 31-40 age group followed by 41-50 age groups. It is lowest in extreme of age. No association was observed between age of the patient and Cytokeratin 5/6 expression in our study. However, Rehim et al., [2] showed an inverse correlation of Cytokeratin 5/6 with the patient's age. Another study results showed, a large number of patients with the positive basal marker expression were belong to the age group of 41 to 50 years, Winter [3]; which is consistent with present study. In present study, among the 41 cases all cases were diagnosed as invasive ductal carcinoma (NST). Other study also found same type of results. In a recent study Atik et al., [4] found that majority of cases were invasive ductal carcinoma $(44.4 \%)$ but the spectrum was wide despite the small number of patients included in their work. Similar to these study Nofech-Mozes et al., [5] and Williams et al., [6] described the vast majority of ductal histology (92\% and 91\%, respectivelly) in their series. There are a number of studies showing the relationship between the histologic grade and hormone negativity in breast carcinomas [7, 8]. Carey et al., [8] examined hormone receptor negative tumors and found that $26 \%$ of cases were triple negative and that these tumors were mainly of high histological grade. Dabbs et al., [9] reviewed morphologically stained 16 triple negative breast carcinomas and reported that all tumors were of high grade according to the Nottingham score. Similar to them all of our triple negative cases were high grade cases constituted the majority of them (75\%). TN carcinomas are highly proliferative breast tumors and could be identified by basal cytokeratin expression. Rakha et al., [7] examined a series of 1944 patients and reported positive immunohistochemical expression of CK5/6 and/or CK14 in 157 (55.7\%) TN cases. Similarly Toyoma et al., [10] examined all their TN breast 52 carcinomas and found $31 \%$ positive for EGFR, 52\% positive for $\mathrm{CK} 5 / 6$ and $55 \%$ positive for CK14. In present study, Out of 41 cases 1 (2. \%) cases were grade 
1, $24(59 \%)$ cases were grade 2 and $16(39 \%)$ were grade 3 . According to the grade and the status of the membrane /cytoplasmic expression of CK 5/6 protein, grade I, grade II and grade III showed $00(00 \%)$, $14(53 \%)$ and $12(46 \%)$ of low and high expression of CK5/6 respectively. After the statistical analysis by SPSS 20 Spearman rank correlation coefficient was $0.99, \mathrm{t}$ value was 43.84 . With a 39 degree of freedom (df $=n-2)$, the $P$ value is $<0.05$. It appears that percentage of CK5/6 expression is higher in high grade of basal like TNBC, which is statistcally significant. This result is consistent with other study results $[11,10$, 7] et al., Another study, performed by Abdelrahman et al., [12] result showed that, morphologically aggressive phenotype of TNBC were grade II-III infiltrative ductal carcinomas. CK5/6 and EGFR expressions were found in $57.1 \%$ and $71.4 \%$ of the cases respectively. However, the combined expression of both CK5/6 and EGFR was observed in $48.6 \%$ of cases. However, Sood and Nigam [11] evaluated 36 cases of TNBC and observed that 61.11\% were CK5/6-positive. Siziopikou et al., [13] investigated CK5/6 and EGFR expressions in 271 patients $(48 ; 18 \%$ of them were TN). Of these cases 32 (67\%) were CK5/6 positive and $22(69 \%)$ EGFR positive. In another study Collins et al., [14] searched the frequency of EGFR and basal cytokeratin expressions in TN breast cancers with or without BRCA-1 mutations and found high rates of positivity, pointing out the association of basal-like phenotype with basal cytokeratins and/or EGFR expression. We recommend doing both $\mathrm{CK} 5 / 6$ and EGFR staining to evaluate TN cases to find better results.

\section{Limitations of the study:}

Study period was of short duration. Small sample size was also a limitation of the present study. So, the results of the study may not reflect the exact picture of the whole country.

\section{CONCLUSION AND RECOMMENDATIONS}

Triple-Negative Breast Cancer' encompasses a heterogeneous group of tumors that possess distinctive pathological and clinical features. Although, a significant overlap was observed between the triplenegative breast cancers and basal like breast carcinoma, the "triple negativity" should not be used as a surrogate marker for the basal-like breast cancers. CK5/6 expression could serve as biomarker for identifying TNBC patients with poor survival. These patients are not benefited from neoadjuvant chemotherapy.

\section{REFERENCES}

1. Kozioł M, Püsküllüoglu M, Zygulska A. PARP inhibitors and their role in the therapy of triplenegative metastatic breast cancer. Przeglad lekarski. 2012 Jan 1; 69(6):265-70.

2. Abd El- Rehim DM, Pinder SE, Paish CE, Bell J, Blamey RW, Robertson JF, Nicholson RI, Ellis IO. Expression of luminal and basal cytokeratins in human breast carcinoma. The Journal of Pathology: A Journal of the Pathological Society of Great Britain and Ireland. 2004 Jun; 203(2):661-71.

3. Winter J. Morphological and immunophenotypic analysis of basal-like carcinoma of the breast. Bioscience Horizons. 2008 Mar 1; 1(1):19-27.

4. Atik E, Guray M, Ozgur T, Canda T. Characterization of immunohistochemical markers in triple negative breast carcinomas. JBUON. 2013 Oct 1; 18(4):88690.

5. Nofech-Mozes S, Trudeau M, Kahn HK, Dent R, Rawlinson E, Sun P, Narod SA, Hanna WM. Patterns of recurrence in the basal and non-basal subtypes of triple-negative breast cancers. Breast cancer research and treatment. 2009 Nov; 118(1):131-7.

6. Williams DJ, Cohen C, Van to T, Page AJ, Lawson D, Sussman ZM, Nassar A. Triple-negative breast carcinoma in women from Vietnam and the United States: characterization of differential marker expression by tissue microarray. Human pathology. 2009 Aug 1; 40(8):1176-81.

7. Rakha EA, El- Sayed ME, Green AR, Lee AH, Robertson JF, Ellis IO. Prognostic markers in triplenegative breast cancer. Cancer. 2007 Jan 1; 109(1):2532.

8. Carey LA, Perou CM, Livasy CA, Dressler LG, Cowan D, Conway K, Karaca G, Troester MA, Tse CK, Edmiston S, Deming SL. Race, breast cancer subtypes, and survival in the Carolina Breast Cancer Study. Jama. 2006 Jun 7; 295(21):2492-502.

9. Bhargava R, Beriwal S, McManus K, Dabbs DJ. CK5 is more sensitive than CK5/6 in identifying the "basallike" phenotype of breast carcinoma. American journal of clinical pathology. 2008 Nov 1; 130(5):724-30.

10. Toyama T, Yamashita H, Kondo N, Okuda K, Takahashi S, Sasaki H, Sugiura H, Iwase H, Fujii Y. Frequently increased epidermal growth factor receptor (EGFR) copy numbers and decreased BRCA1 mRNA expression in Japanese triple-negative breast cancers. BMC cancer. 2008 Dec; 8(1):1-2.

11. Sood N, Nigam JS. Correlation of CK5 and EGFR with clinicopathological profile of triple-negative breast cancer. Pathology research international. 2014; 2014.

12. Abdelrahman AE, Rashed HE, Abdelgawad M, Abdelhamid MI. Prognostic impact of EGFR and cytokeratin 5/6 immunohistochemical expression in triple-negative breast cancer. Annals of diagnostic pathology. 2017 Jun 1; 28:43-53.

13. Siziopikou KP, Cobleigh M. The basal subtype of breast carcinomas may represent the group of breast tumors that could benefit from EGFR-targeted therapies. The Breast. 2007 Feb 1; 16(1):104-7.

14. Collins LC, Martyniak A, Kandel MJ, Stadler ZK, Masciari S, Miron A, Richardson AL, Schnitt SJ, Garber JE. Basal cytokeratin and epidermal growth factor receptor expression are not predictive of BRCA1 mutation status in women with triplenegative breast cancers. The American journal of surgical pathology. 2009 Jul 1; 33(7):1093-7. 